International Research Journal of Engineering, IT \& Scientific Research
Available online at https://sloap.org/journals/index.php/irjeis/
Vol. 5 No. 3 May 2019, pages: $28 \sim 41$
ISSN: 2454-2261
https://doi.org/10.21744/irjeis.v5n3.645

\title{
Effect of Reversing Lamination Scheme
}

Osama Mohammed Elmardi Suleiman a Mahmoud Yassin Osman ${ }^{\text {b }}$ Tagelsir Hassan $^{c}$

Article history:

Received: 27 December 2018

Accepted: 31 March 2019

Published: 31 May 2019

\section{Keywords:}

classical laminated plate theory; composite laminated decks

plates;

finite element;

Fortran program;

reversing lamination scheme;

\begin{abstract}
New numerical results are generated for in-plane compressive biaxial buckling which serves to quantify the effect of reversing lamination scheme on buckling loading. The buckling loads have been determined for two modulus ratios 40 and 5. It is observed that the buckling loads are completely the same for the given first three modes. Therefore, it can be concluded that the buckling load of laminated plates will remain the same even if the lamination order is reversed. The reason behind this is that the transformed elastic coefficients, $\left[C_{i j}\right]$, are equal for both lamination schemes.
\end{abstract}

\section{Author correspondence:}

Osama Mohammed Elmardi Suleiman,

Nile Valley University, (Sudan/East Africa).

Email address: osamamm64@gmail.com

\section{Introduction}

The effects of lamination scheme on the non - dimensional critical buckling loads of laminated composite plates are investigated.

The material chosen has the following properties: $E_{1} / E_{2}=5,10,20,25,40 ; G_{12}=G_{13}=G_{23}=0.5 E_{2} ; v_{12}=$ 0.25 .

Several numerical methods could be used in this study, but the main ones are finite difference method (FDM), dynamic relaxation coupled with finite difference method (DR) as is shown in references (Turvey \& Osman, 1990; Turvey \& Osman, 1989); Turvey \& Osman, 1991; Elmardi, 2014; 2015; 2015a; 2015b; 2016), and finite element method (FEM).

In the present work, a numerical method known as the finite element method (FEM) is used. It is a numerical procedure for obtaining solutions to many of the problems encountered in engineering analysis. It has two primary subdivisions. The first utilizes discrete elements to obtain the joint displacements and member forces of a structural

${ }^{a}$ Nile Valley University, (Sudan/East Africa)

${ }^{\mathrm{b}}$ Kassala University, (Sudan/East Africa)

c Omdurman Islamic University, (Sudan/East Africa) 
framework. The second uses the continuum elements to obtain approximate solutions to heat transfer, fluid mechanics, and solid mechanics problem. The formulation using the discrete element is referred to as matrix analysis of structures and yields results identical with the classical analysis of structural frameworks. The second approach is the true finite element method. It yields approximate values of the desired parameters at specific points called nodes. A general finite element computers program, however, is capable of solving both types of problems and the name" finite element method" is often used to denote both the discrete element and the continuum element formulations.

The finite element method combines several mathematical concepts to produce a system of linear and non linear equations. The number of equations is usually very large, anywhere from 20 to 20,000 or more and requires the computational power of the digital computer.

It is impossible to document the exact origin of the finite element method because the basic concepts have evolved over a period of 150 or more years. The method as we know it today is an outgrowth of several papers published in the $1950^{\text {th }}$ that extended the matrix analysis of structures to continuum bodies. The space exploration of the $1960^{\text {th }}$ provided money for basic research, which placed the method of a firm mathematical foundation and stimulated the development of multi-purpose computer programs that implemented the method. The design of airplanes, unmanned drones, missiles, space capsules, and the like, provided application areas.

\section{Materials and Methods}

The finite element method (FEM) is a powerful numerical method, which is used as a computational technique for the solution of differential equations that arise in various fields of engineering and applied sciences. The finite element method is based on the concept that one can replace any continuum by an assemblage of simply shaped elements, called finite elements with well-defined force, displacement, and material relationships. While one may not be able to derive a closed - form solution for the continuum, one can derive approximate solutions for the element assemblage that replaces it. The approximate solutions or approximation functions are often constructed using ideas from interpolation theory, and hence they are also called interpolation functions. For more details refer to References (Larry, 1984; Al - khafaji \& Tooley, 1986; McConkey, 1993).

\section{Results and Discussions}

\subsection{Mathematical Formulations}

1) Introduction

The following assumptions were made in developing the mathematical formulations of laminated plates:

a) All layers behave elastically;

b) Displacements are small compared with the plate thickness;

c) Perfect bonding exists between layers;

d) The laminate is equivalent to a single anisotropic layer;

e) The plate is flat and has a constant thickness;

f) The plate buckles in a vacuum and all kinds of damping are neglected.

Unlike homogeneous plates, where the coordinates are chosen solely based on the plate shape, coordinates for laminated plates should be chosen carefully. There are two main factors for the choice of the coordinate system. The first factor is the shape of the plate. Where rectangular plates will be best represented by the choice of rectangular (i.e. Cartesian) coordinates. It will be relatively easy to represent the boundaries of such plates with coordinates. The second factor is the fiber orientation or orthotropic. If the fibers are set straight within each lamina, then rectangular orthotropic would result. It is possible to set the fibers in a radial and circular fashion, which would result in circular orthotropic. Indeed, the fibers can also be set in elliptical directions, which would result in elliptical orthotropic.

The choice of the coordinate system is of critical importance for laminated plates. This is because plates with rectangular orthotropic could be set on rectangular, triangular, circular or other boundaries. Composite materials with rectangular orthotropic are the most popular, mainly because of their ease in design and manufacturing. The equations that follow are developed for materials with rectangular orthotropic.

Suleiman, O. M. E., Osman, M. Y., \& Hassan, T. (2019). Effect of reversing lamination scheme. International Research Journal of Engineering, IT \& Scientific Research, 5(3), 28-42. https://doi.org/10.21744/irjeis.v5n3.645 
Figure 1 shows the geometry of a plate with rectangular orthotropic drawn in the cartesian coordinates $\mathrm{X}$, Y, and Z or 1,2, and 3. The parameters used in such a plate are: (1) the length in the X-direction, (a); (2) the length in the Y direction (i.e. breadth), (b); and (3) the length in the $\mathrm{Z}$-direction (i.e. thickness), (h).

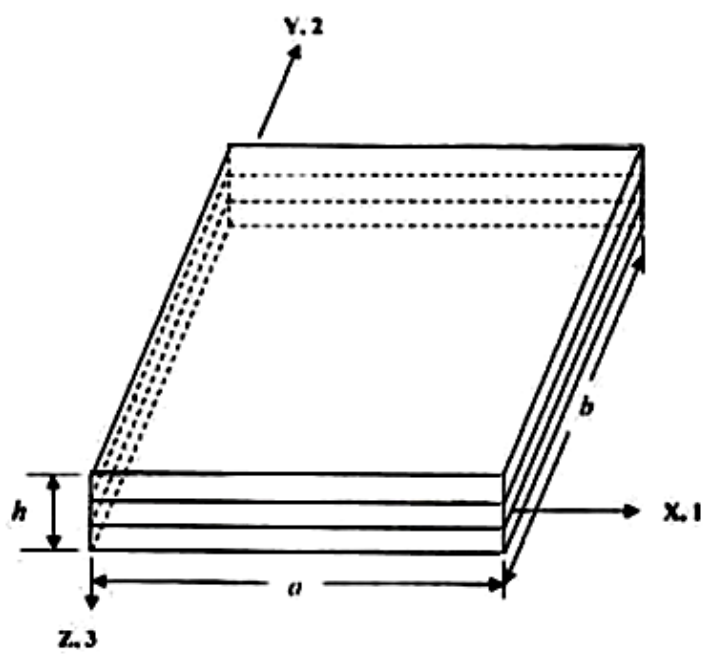

Figure 1. The geometry of a laminated composite plate

2) Fundamental Equations of Elasticity

Classical laminated plate theory (CLPT) is selected to formulate the problem. Consider a thin plate of length a, breadth $\mathrm{b}$, and thickness has shown in Figure2(a), subjected to in-plane loads Rx, Ry and Rxy as shown in Figure2(b). The in-plane displacements $\boldsymbol{u}(\boldsymbol{x}, \boldsymbol{y}, \boldsymbol{z})$ and $\boldsymbol{v}(\boldsymbol{x}, \boldsymbol{y}, \boldsymbol{z})$ can be expressed in terms of the out of plane displacement $\boldsymbol{w}(\boldsymbol{x}, \boldsymbol{y})$ as shown below:

The displacements are:

$$
\left.\begin{array}{l}
u(x, y, z)=u_{o}(x, y)-z \frac{\partial w}{\partial x} \\
v(x, y, z)=v_{o}(x, y)-z \frac{\partial w}{\partial y} \\
w(x, y, z)=w_{o}(x, y)
\end{array}\right\}
$$

Where $u_{o}, v_{o}$ and $w_{o}$ are mid - plane displacements in the direction of the $x, y$ and $z$ axes respectively; $z$ is the perpendicular distance from mid - plane to the layer plane.

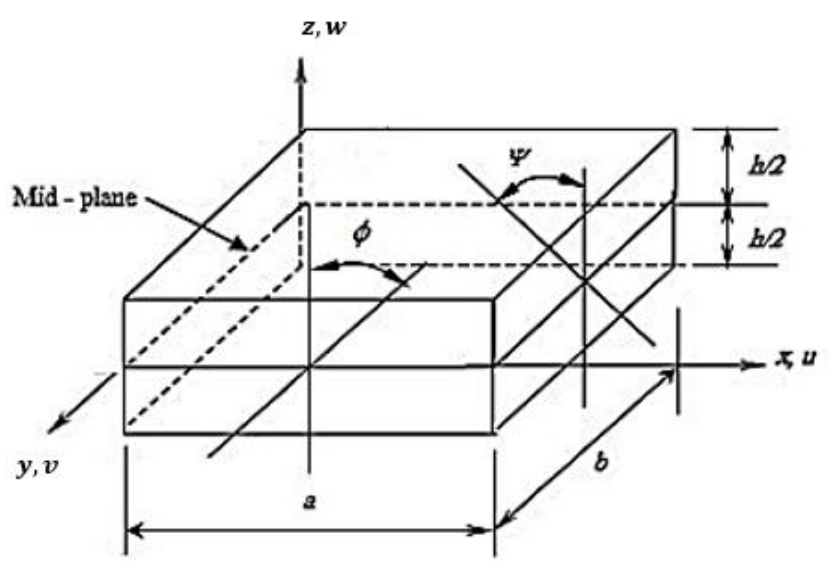

( a ) 


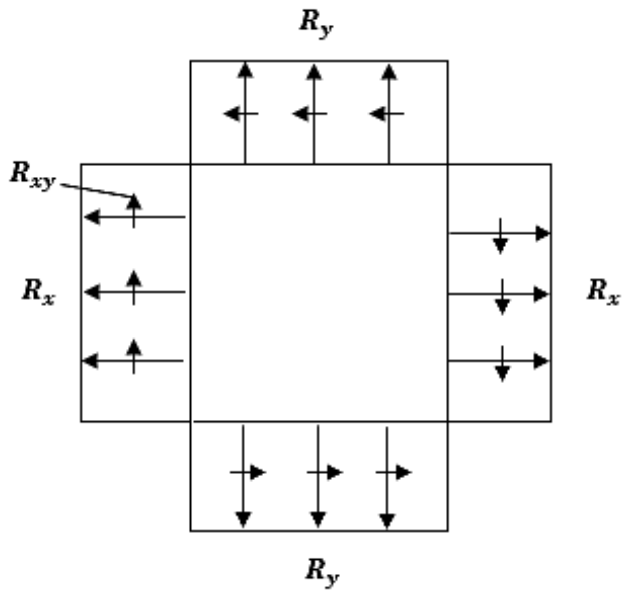

( b )

Figure 2. A plate showing dimensions and deformations

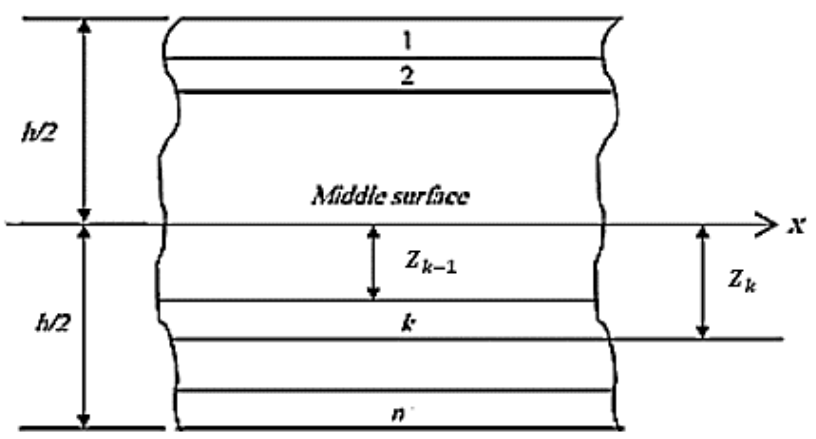

Figure 3. The geometry of an n-layered laminate

The plate is shown in Figure 2(a) is constructed of an arbitrary number of orthotropic layers bonded together as in Figure 3.

The strains are:

$$
\left.\begin{array}{l}
\epsilon_{x}=\frac{\partial u_{o}}{\partial x}-z \frac{\partial^{2} w}{\partial x^{2}}+\frac{1}{2}\left(\frac{\partial w}{\partial x}\right)^{2} \\
\epsilon_{y}=\frac{\partial v_{o}}{\partial y}-z \frac{\partial^{2} w}{\partial y^{2}}+\frac{1}{2}\left(\frac{\partial w}{\partial y}\right)^{2} \\
\gamma=\frac{\partial v_{o}}{\partial x}+\frac{\partial u_{o}}{\partial y}-2 z \frac{\partial^{2} w}{\partial x \partial y}+\left(\frac{\partial w}{\partial x}\right)\left(\frac{\partial w}{\partial y}\right)
\end{array}\right\}
$$

The virtual strains:

Suleiman, O. M. E., Osman, M. Y., \& Hassan, T. (2019). Effect of reversing lamination scheme. International Research Journal of Engineering, IT \& Scientific Research, 5(3), 28-42. https://doi.org/10.21744/irjeis.v5n3.645 


$$
\begin{gathered}
\delta \epsilon_{x}=\frac{\partial}{\partial x} \delta u_{o}-z \frac{\partial^{2}}{\partial x^{2}} \delta w+\frac{\partial w}{\partial x} \frac{\partial}{\partial x} \delta w \\
\delta \epsilon_{y}=\frac{\partial}{\partial y} \delta v_{o}-z \frac{\partial^{2}}{\partial y^{2}} \delta w+\frac{\partial w}{\partial y} \frac{\partial}{\partial y} \delta w \\
\delta \gamma=\frac{\partial}{\partial x} \delta v_{o}+\frac{\partial}{\partial y} \delta u_{o}-2 z \frac{\partial^{2}}{\partial x \partial y} \delta w \\
+\frac{\partial w}{\partial x} \frac{\partial}{\partial y} \delta w+\frac{\partial}{\partial x} \delta w \frac{\partial w}{\partial y}
\end{gathered}
$$

The virtual strain energy:

$$
\delta U=\int_{V} \delta \epsilon^{T} \sigma d V
$$

But,

$$
\sigma=C \epsilon
$$

Where,

$$
C=C_{i j}(i, j=1,2,6)
$$

$$
\therefore \delta U=\int_{V} \delta \epsilon^{T} C \delta \epsilon d V
$$

If we neglect the in-plane displacements $u_{o}$ and $v_{o}$ and considering only the linear terms in the strain - displacement equations, we write:

$$
\delta \epsilon=-z\left|\begin{array}{c}
\frac{\partial^{2}}{\partial x^{2}} \\
\frac{\partial^{2}}{\partial y^{2}} \\
2 \frac{\partial^{2}}{\partial x \partial y}
\end{array}\right| \delta w
$$

\subsection{The Numerical Method}

The finite element is used in this analysis as a numerical method to predict the buckling loads and shape modes of buckling of laminated rectangular plates ( Suleiman, 2016). In this method of analysis, four - the noded type of elements is chosen. These elements are the four - noded bilinear rectangular elements of a plate. Each element has three degrees of freedom at each node. The degrees of freedom are the lateral displacement $(w)$, and the rotations $(\phi)$ and $(\psi)$ about the $(X)$ and $(Y)$ axes respectively.

The finite element method is formulated by the energy method.

Now express $w$ in terms of the shape functions $N$ and noded displacements $a^{e}$, equation (6) can be written as:

Where,

$$
\delta \epsilon=-z B \delta a^{e}
$$

and

$$
B^{T}=\left[\begin{array}{lll}
\frac{\partial^{2} N_{i}}{\partial x^{2}} & \frac{\partial^{2} N_{i}}{\partial y^{2}} & 2 \frac{\partial^{2} N_{i}}{\partial x \partial y}
\end{array}\right]
$$

The stress - strain relation is:

$$
N_{i} a_{i}^{e}=\left[w_{i}\right] \quad i=1, n
$$

$$
\sigma=C \epsilon
$$

Where $C$ are the material properties which could be written as follows:

$$
C=\left[\begin{array}{lll}
C_{11} & C_{12} & C_{16} \\
C_{12} & C_{22} & C_{26} \\
C_{16} & C_{26} & C_{66}
\end{array}\right]
$$


Where $V$ denotes volume.

$$
\delta U=\int_{V}\left(B \delta a^{e}\right)^{T}\left(C z^{2}\right) B a^{e} d V
$$

$$
\delta U=\delta a^{e T} \int_{V} B^{T} D B a^{e} d x d y=\delta a^{e T} K^{e} a^{e}
$$

Where $D_{i j}=\sum_{k=1}^{n} \int_{Z_{k-1}}^{Z_{k}} C_{i j} Z^{2} d Z$ is the bending stiffness, and $K^{e}$ is the element stiffness matrix which could be written as follows:

$$
K^{e}=\int B^{T} D B d x d y
$$

The virtual work done by external forces can be expressed as follows: Refer to Figure4. Denoting the nonlinear part of strain by $\delta \epsilon^{\prime}$

Where

$$
\delta W=\iint \delta \epsilon^{\prime T} \sigma^{\prime} d V=\int \delta \epsilon^{\prime T} N d x d y
$$

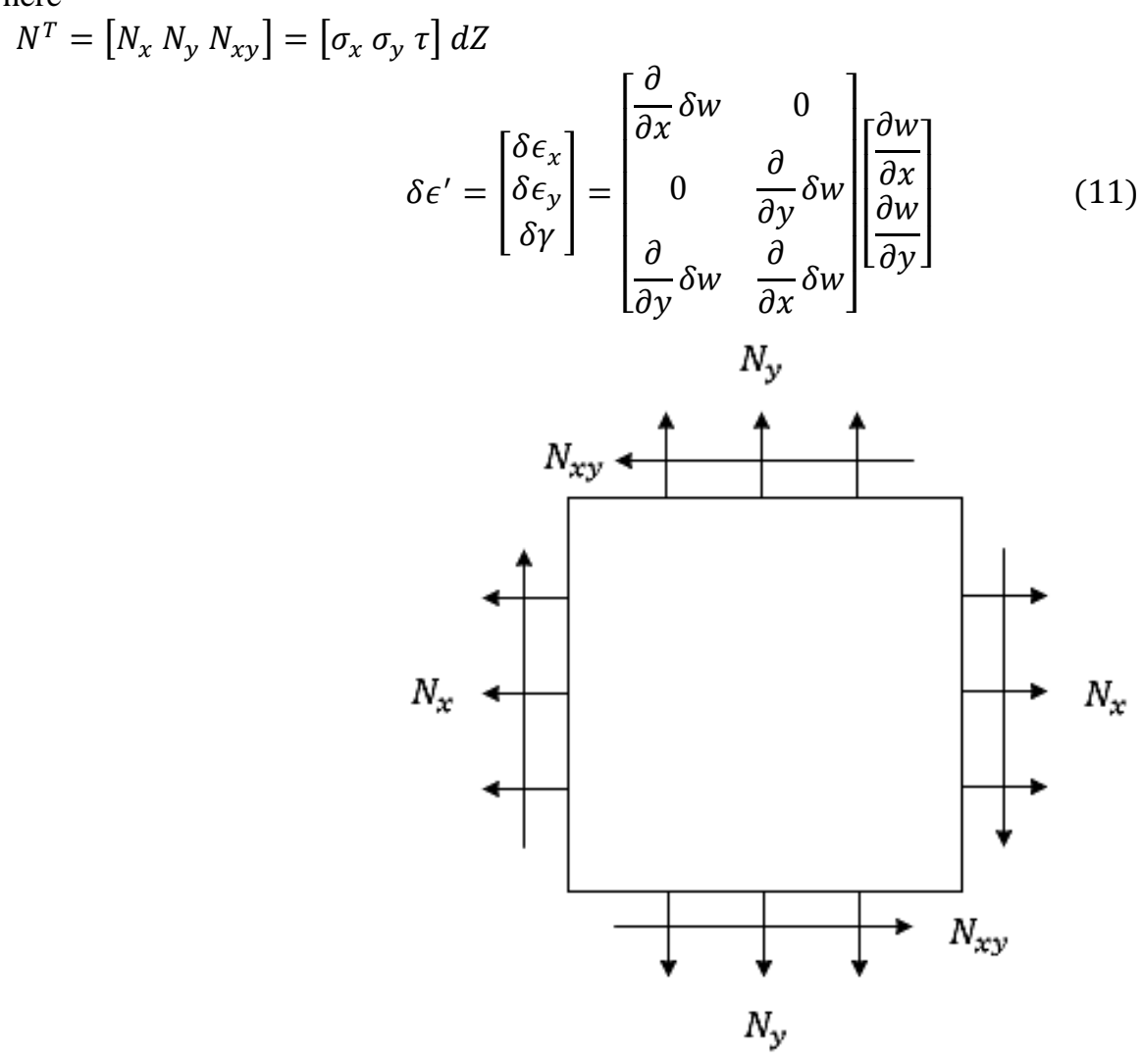

Figure 4. External forces acting on an element

Hence,

This can be written as:

$$
\delta W=\iint\left[\begin{array}{l}
\frac{\partial w}{\partial x} \\
\frac{\partial w}{\partial y}
\end{array}\right]^{T}\left[\begin{array}{ccc}
\frac{\partial}{\partial x} \delta w & 0 & \frac{\partial}{\partial y} \delta w \\
0 & \frac{\partial}{\partial y} \delta w & \frac{\partial}{\partial x} \delta w
\end{array}\right]\left[\begin{array}{c}
N_{x} \\
N_{y} \\
N_{x y}
\end{array}\right] d x d y
$$

Suleiman, O. M. E., Osman, M. Y., \& Hassan, T. (2019). Effect of reversing lamination scheme. International Research Journal of Engineering, IT \& Scientific Research, 5(3), 28-42. https://doi.org/10.21744/irjeis.v5n3.645 
Now $w=N_{i} a_{i}^{e}$

$$
\delta W=\iint\left[\begin{array}{l}
\frac{\partial}{\partial x} \delta w \\
\frac{\partial}{\partial y} \delta w
\end{array}\right]^{T}\left[\begin{array}{cc}
N_{x} & N_{x y} \\
N_{x y} & N_{y}
\end{array}\right]\left[\begin{array}{l}
\frac{\partial w}{\partial x} \\
\frac{\partial w}{\partial y}
\end{array}\right] d x d y
$$

$$
\delta W=\delta a^{e T} \iint\left[\begin{array}{c}
\frac{\partial N_{i}}{\partial x} \\
\frac{\partial N_{i}}{\partial y}
\end{array}\right]^{T}\left[\begin{array}{cc}
N_{x} & N_{x y} \\
N_{x y} & N_{y}
\end{array}\right]\left[\begin{array}{c}
\frac{\partial N_{i}}{\partial x} \\
\frac{\partial N_{i}}{\partial y}
\end{array}\right] a^{e} d x d y
$$

Substitute $P_{x}=-N_{x}, P_{y}=-N_{y}, P_{x y}=-N_{x y}$

$$
\delta W=-\delta a^{e T} \iint\left[\begin{array}{c}
\frac{\partial N_{i}}{\partial x} \\
\frac{\partial N_{i}}{\partial y}
\end{array}\right]^{T}\left[\begin{array}{cc}
P_{x} & P_{x y} \\
P_{x y} & P_{y}
\end{array}\right]\left[\begin{array}{c}
\frac{\partial N_{i}}{\partial x} \\
\frac{\partial N_{i}}{\partial y}
\end{array}\right] a^{e} d x d y
$$

Therefore, equation (15) could be written in the following form:

Where,

$$
\delta W=-\delta a^{e T} K^{D} a^{e}
$$

$$
K^{D}=\iint\left[\begin{array}{c}
\frac{\partial N_{i}}{\partial x} \\
\frac{\partial N_{i}}{\partial y}
\end{array}\right]^{T}\left[\begin{array}{cc}
P_{x} & P_{x y} \\
P_{x y} & P_{y}
\end{array}\right]\left[\begin{array}{c}
\frac{\partial N_{i}}{\partial x} \\
\frac{\partial N_{i}}{\partial y}
\end{array}\right] d x d y
$$

$K^{D}$ is the differential stiffness matrix known also as geometric stiffness matrix, initial stress matrix, and initial load matrix.

The total energy:

$$
\delta U+\delta W=0
$$

Since $\delta a^{e}$ is an arbitrary displacement which is not zero, then

$$
K^{e} a^{e}-K^{D} a^{e}=0
$$

Now let us compute the elements stiffness and the differential matrices.

$$
\begin{gathered}
K^{e}=\iint B^{T} D B d x d y \\
K^{e}=\iint\left[\begin{array}{c}
\frac{\partial^{2} N_{i}}{\partial x^{2}} \\
\frac{\partial^{2} N_{i}}{\partial y^{2}} \\
2 \frac{\partial^{2} N_{i}}{\partial x \partial y}
\end{array}\right]^{T}\left[\begin{array}{lll}
D_{11} & D_{12} & D_{16} \\
D_{12} & D_{22} & D_{26} \\
D_{16} & D_{26} & D_{66}
\end{array}\right]\left[\begin{array}{c}
\frac{\partial^{2} N_{i}}{\partial x^{2}} \\
\frac{\partial^{2} N_{i}}{\partial y^{2}} \\
2 \frac{\partial^{2} N_{i}}{\partial x \partial y}
\end{array}\right] d x d y
\end{gathered}
$$

The elements of stiffness matrix can be expressed as follows:

$$
\begin{gathered}
K_{i j}^{e}=\iint\left[D_{11} \frac{\partial^{2} N_{i}}{\partial x^{2}} \frac{\partial^{2} N_{j}}{\partial x^{2}}+D_{12}\left(\frac{\partial^{2} N_{i}}{\partial y^{2}} \frac{\partial^{2} N_{j}}{\partial x^{2}}+\frac{\partial^{2} N_{i}}{\partial x^{2}} \frac{\partial^{2} N_{j}}{\partial y^{2}}\right)+2 D_{16}\left(\frac{\partial^{2} N_{i}}{\partial x \partial y} \frac{\partial^{2} N_{j}}{\partial x^{2}}+\frac{\partial^{2} N_{i}}{\partial x^{2}} \frac{\partial^{2} N_{j}}{\partial x \partial y}\right)+D_{22} \frac{\partial^{2} N_{i}}{\partial y^{2}} \frac{\partial^{2} N_{j}}{\partial y^{2}}\right. \\
\left.+2 D_{26}\left(\frac{\partial^{2} N_{i}}{\partial x \partial y} \frac{\partial^{2} N_{j}}{\partial y^{2}}+\frac{\partial^{2} N_{i}}{\partial y^{2}} \frac{\partial^{2} N_{j}}{\partial x \partial y}\right)+4 D_{66} \frac{\partial^{2} N_{i}}{\partial x \partial y} \frac{\partial^{2} N_{j}}{\partial x \partial y}\right] d x d y \text { (19) }
\end{gathered}
$$

The elements differential stiffness matrix can be expressed as follows;

$$
K_{i j}^{D}=\iint\left[P_{x} \frac{\partial N_{i}}{\partial x} \frac{\partial N_{j}}{\partial x}+P_{x y}\left(\frac{\partial N_{i}}{\partial y} \frac{\partial N_{j}}{\partial x}+\frac{\partial N_{i}}{\partial x} \frac{\partial N_{j}}{\partial y}\right)+P_{y} \frac{\partial N_{i}}{\partial y} \frac{\partial N_{j}}{\partial y}\right] d x d y
$$

The shape local coordinate for a 4 - noded element is shown below in Figure 5 


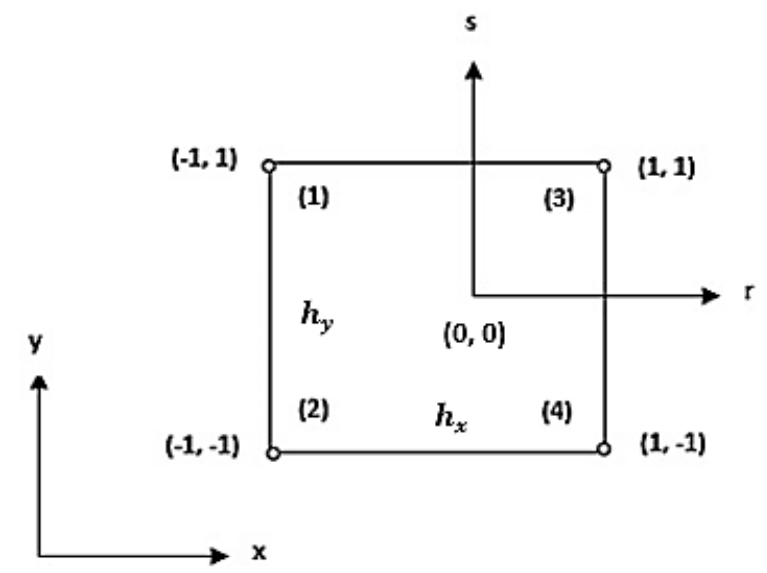

Figure 5. A four noded element with local and global co - ordinates

The shape functions for the 4 - noded element expressed in global co - ordinates $(x, y)$ are as follows:

Where,

$$
\begin{aligned}
& w=N_{1} w_{1}+N_{2} \phi_{1}+N_{3} \psi_{1}+N_{4} w_{2}+N_{5} \phi_{2}+N_{6} \psi_{2} \\
& +N_{7} w_{3}+N_{8} \phi_{3}+N_{9} \psi_{3}+N_{10} w_{4}+N_{11} \phi_{4}+N_{12} \psi_{4}
\end{aligned}
$$

$$
\phi=\frac{\partial w}{\partial x}, \quad \psi=\frac{\partial w}{\partial y}
$$

The shape functions in local co - ordinates are as follows:

$$
\begin{gathered}
N_{i}=a_{i 1}+a_{i 2} r+a_{i 3} s+a_{i 4} r^{2}+a_{i 5} r s+a_{i 6} s^{2}+a_{i 7} r^{3}+a_{i 8} r^{2} s+a_{i 9} r s^{2} \\
+a_{i 10} s^{3}+a_{i 11} r^{3} s+a_{i 12} r s^{3} \\
\begin{array}{c}
N_{j}=a_{j 1}+a_{j 2} r+a_{j 3} s \\
+a_{j 4} r^{2}+a_{j 5} r s+a_{j 6} s^{2}+a_{j 7} r^{3}+a_{j 8} r^{2} s+a_{j 9} r s^{2} \\
+a_{j 10} s^{3}+a_{j 11} r^{3} s+a_{j 12} r s^{3}
\end{array}
\end{gathered}
$$

The integrals of the shape functions in local co - ordinates are as follows:

$$
\begin{aligned}
& q_{1}=\iint \frac{\partial^{2} N_{i}}{\partial r^{2}} \frac{\partial^{2} N_{j}}{\partial r^{2}} d r d s=16\left[a_{i 4} a_{j 4}+3 a_{i 7} a_{j 7}+\frac{1}{3} a_{i 8} a_{j 8}+a_{i 11} a_{j 11}\right] \\
& q_{2}=\iint \frac{\partial^{2} N_{i}}{\partial s^{2}} \frac{\partial^{2} N_{j}}{\partial s^{2}} d r d s=16\left[a_{i 6} a_{j 6}+\frac{1}{3} a_{i 9} a_{j 9}+3 a_{i 10} a_{j 10}+a_{i 12} a_{j 12}\right] \\
& q_{3}=\iint \frac{\partial^{2} N_{i}}{\partial r^{2}} \frac{\partial^{2} N_{j}}{\partial s^{2}} d r d s=16\left[a_{i 4} a_{j 6}+a_{i 7} a_{j 9}+a_{i 8} a_{j 10}+a_{i 11} a_{j 12}\right] \\
& q_{4}=\iint \frac{\partial^{2} N_{i}}{\partial s^{2}} \frac{\partial^{2} N_{j}}{\partial r^{2}} d r d s=16\left[a_{i 6} a_{j 4}+a_{i 9} a_{j 7}+a_{i 10} a_{j 8}+a_{i 12} a_{j 11}\right] \\
& q_{5}=\iint \frac{\partial^{2} N_{i}}{\partial r^{2}} \frac{\partial^{2} N_{j}}{\partial r \partial s} d r d s=8\left[a_{i 4} a_{j 5}+a_{i 4} a_{j 11}+2 a_{i 7} a_{j 8}+a_{i 4} a_{j 12}\right. \\
& \left.+\frac{2}{3} a_{i 8} a_{j 9}\right] \\
& q_{6}=\iint \frac{\partial^{2} N_{i}}{\partial r \partial s} \frac{\partial^{2} N_{j}}{\partial r^{2}} d r d s=8\left[a_{i 5} a_{j 4}+2 a_{i 8} a_{j 7}+a_{i 11} a_{j 4}+\frac{2}{3} a_{i 9} a_{j 8}\right. \\
& \left.+a_{i 12} a_{j 4}\right] \\
& q_{7}=\iint \frac{\partial^{2} N_{i}}{\partial s^{2}} \frac{\partial^{2} N_{j}}{\partial r \partial s} d r d s=8\left[a_{i 6} a_{j 5}+a_{i 6} a_{j 11}+\frac{2}{3} a_{i 9} a_{j 8}\right] \\
& q_{8}=\iint \frac{\partial^{2} N_{i}}{\partial r \partial s} \frac{\partial^{2} N_{j}}{\partial s^{2}} d r d s=8\left[a_{i 5} a_{j 6}+\frac{2}{3} a_{i 8} a_{j 9}+a_{i 11} a_{j 6}\right]
\end{aligned}
$$

Suleiman, O. M. E., Osman, M. Y., \& Hassan, T. (2019). Effect of reversing lamination scheme. International Research Journal of Engineering, IT \& Scientific Research, 5(3), 28-42. 


$$
\begin{aligned}
& q_{9}=\iint \frac{\partial^{2} N_{i}}{\partial r \partial s} \frac{\partial^{2} N_{j}}{\partial r \partial s} d r d s=4\left[a_{i 5} a_{j 5}+a_{i 5} a_{j 11}+\frac{4}{3} a_{i 8} a_{j 8}+a_{i 5} a_{j 12}\right. \\
& \left.+\frac{4}{3} a_{i 9} a_{j 9}+a_{i 11} a_{j 12}+a_{i 12} a_{j 11}+\frac{9}{5} a_{i 12} a_{j 12}\right] \\
& q_{10}=\iint \frac{\partial N_{i}}{\partial r} \frac{\partial N_{j}}{\partial r} d r d s=4\left[a_{i 2} a_{j 2}+\frac{1}{3}\left(3 a_{i 2} a_{j 7}+4 a_{i 4} a_{j 4}+3 a_{i 7} a_{j 2}\right.\right. \\
& +a_{i 7} a_{j 9}+a_{i 5} a_{j 5}+a_{i 9} a_{j 2}+a_{i 5} a_{j 11}+a_{i 7} a_{j 9}+\frac{4}{3} a_{i 8} a_{j 8}+a_{i 9} a_{j 7} \\
& \left.a_{i 11} a_{j 5}\right)+\frac{1}{5}\left(a_{i 5} a_{j 12}+a_{i 9} a_{j 9}+a_{i 12} a_{j 5}+9 a_{i 7} a_{j 7}+3 a_{i 11} a_{j 11}+a_{i 11} a_{j 12}\right. \\
& \left.\left.+a_{i 12} a_{j 11}\right)+\frac{1}{7} a_{i 12} a_{j 12}\right] \\
& q_{11}=\iint \frac{\partial N_{i}}{\partial s} \frac{\partial N_{j}}{\partial s} d r d s=4\left[a_{i 3} a_{j 3}+\frac{1}{3}\left(a_{i 3} a_{j 8}+a_{i 5} a_{j 5}+a_{i 8} a_{j 3}\right.\right. \\
& +3 a_{i 3} a_{j 10}+4 a_{i 6} a_{j 6}+3 a_{i 10} a_{j 3}+a_{i 5} a_{j 12}+a_{i 8} a_{j 10}+\frac{4}{3} a_{i 9} a_{j 9}+a_{i 10} a_{j 8} \\
& \left.+a_{i 12} a_{j 5}\right)+\frac{1}{5}\left(a_{i 5} a_{j 11}+a_{i 8} a_{j 8}+a_{i 11} a_{j 5}+9 a_{i 10} a_{j 10}+a_{i 11} a_{j 12}+a_{i 12} a_{j 11}\right. \\
& \left.\left.+3 a_{i 2} a_{j 12}\right)+\frac{1}{7} a_{i 11} a_{j 11}\right] \\
& q_{12}=\iint \frac{\partial N_{i}}{\partial r} \frac{\partial N_{j}}{\partial s} d r d s=4\left[a_{i 2} a_{j 3}+\frac{1}{3}\left(a_{i 2} a_{j 8}+2 a_{i 4} a_{j 5}+3 a_{i 7} a_{j 8}\right.\right. \\
& +3 a_{i 2} a_{j 10}+2 a_{i 5} a_{j 6}+a_{i 9} a_{j 3}+2 a_{i 4} a_{j 12}+3 a_{i 7} a_{j 10}+\frac{4}{3} a_{i 8} a_{j 9}+\frac{1}{3} a_{i 9} a_{j 8} \\
& \left.\left.+2 a_{i 11} a_{j 6}\right)\right] \\
& q_{13}=\iint \frac{\partial N_{i}}{\partial s} \frac{\partial N_{j}}{\partial r} d r d s=4\left[a_{i 3} a_{j 2}+\frac{1}{3}\left(3 a_{i 3} a_{j 7}+2 a_{i 5} a_{j 4}+a_{i 8} a_{j 2}\right.\right. \\
& +a_{i 3} a_{j 9}+2 a_{i 6} a_{j 5}+3 a_{i 10} a_{j 2}+2 a_{i 6} a_{j 11}+\frac{1}{3} a_{i 8} a_{j 9}+\frac{4}{3} a_{i 9} a_{j 8}+3 a_{i 10} a_{j 7} \\
& \left.\left.+2 a_{i 12} a_{j 4}\right)+\frac{1}{5}\left(2 a_{i 6} a_{j 12}+3 a_{i 10} a_{j 9}+3 a_{i 8} a_{j 7}+2 a_{i 11} a_{j 4}\right)\right]
\end{aligned}
$$

The values of the integrals are converted from local co - ordinate $(r, s)$ to global co - ordinates.

The integrals of the shape functions in global co - ordinates are as follows:

$$
\begin{aligned}
& r_{1}=\iint \frac{\partial^{2} N_{i}}{\partial x^{2}} \frac{\partial N_{j}}{\partial x^{2}} d x d y=\left(\frac{4 h_{y}}{h_{x}^{3}}\right) q_{1}=\frac{4 n^{3} b}{m a^{3}} q_{1} \\
& r_{2}=\iint \frac{\partial^{2} N_{i}}{\partial y^{2}} \frac{\partial^{2} N_{j}}{\partial y^{2}} d x d y=\left(\frac{4 h_{x}}{h_{y}^{3}}\right) q_{2}=\frac{4 a m^{3}}{n b^{3}} q_{2} \\
& r_{3}=\iint \frac{\partial^{2} N_{i}}{\partial x^{2}} \frac{\partial^{2} N_{j}}{\partial y^{2}} d x d y=\left(\frac{4}{h_{y} h_{x}}\right) q_{3}=\frac{4 m n}{a b} q_{3} \\
& r_{4}=\iint \frac{\partial^{2} N_{i}}{\partial y^{2}} \frac{\partial^{2} N_{j}}{\partial x^{2}} d x d y=\left(\frac{4}{h_{y} h_{x}}\right) q_{4}=\frac{4 m n}{a b} q_{4} \\
& r_{5}=\iint \frac{\partial^{2} N_{i}}{\partial x^{2}} \frac{\partial^{2} N_{j}}{\partial x \partial y} d x d y=\left(\frac{4}{h_{x}^{2}}\right) q_{5}=\frac{4 n^{2}}{a^{2}} q_{5} \\
& r_{6}=\iint \frac{\partial^{2} N_{i}}{\partial x \partial y} \frac{\partial^{2} N_{j}}{\partial x^{2}} d x d y=\left(\frac{4}{h_{x}^{2}}\right) q_{6}=\frac{4 n^{2}}{a^{2}} q_{6} \\
& r_{7}=\iint \frac{\partial^{2} N_{i}}{\partial y^{2}} \frac{\partial^{2} N_{j}}{\partial x \partial y} d x d y=\left(\frac{4}{h_{y}^{2}}\right) q_{7}=\frac{4 m^{2}}{a^{2}} q_{7} \\
& r_{8}=\iint \frac{\partial^{2} N_{i}}{\partial x \partial y} \frac{\partial^{2} N_{j}}{\partial y^{2}} d x d y=\left(\frac{4}{h_{y}^{2}}\right) q_{8}=\frac{4 m^{2}}{b^{2}} q_{8}
\end{aligned}
$$




$$
\begin{aligned}
& r_{9}=\iint \frac{\partial^{2} N_{i}}{\partial x \partial y} \frac{\partial^{2} N_{j}}{\partial x \partial y} d x d y=\left(\frac{4}{h_{y} h_{x}}\right) q_{9}=\frac{4 m n}{a b} q_{9} \\
& r_{10}=\iint \frac{\partial N_{i}}{\partial x} \frac{\partial N_{j}}{\partial x} d x d y=\left(\frac{h_{y}}{h_{x}}\right) q_{10}=\frac{b n}{a m} q_{10} \\
& r_{11}=\iint \frac{\partial N_{i}}{\partial y} \frac{\partial N_{j}}{\partial y} d x d y=\left(\frac{h_{x}}{h_{y}}\right) q_{11}=\frac{a m}{b n} q_{11} \\
& r_{12}=\iint \frac{\partial N_{i}}{\partial x} \frac{\partial N_{j}}{\partial y} d x d y=q_{12} \\
& r_{13}=\iint \frac{\partial N_{i}}{\partial y} \frac{\partial N_{j}}{\partial x} d x d y=q_{13}
\end{aligned}
$$

In the previous equations $h_{x}=\frac{a}{n}$ and $h_{y}=\frac{b}{m}$ where $a$ and $b$ are the lengths of the plate along the $x-$ and $y-$ axis respectively. $n$ and $m$ are the number of elements in the $x$-and $y$-directions respectively.

The elements of the stiffness matrix and the differential matrix can be written as follows:

$K_{i j}=D_{11} r_{1}+D_{12} r_{4}+2 D_{16} r_{3}+D_{12} r_{3}+D_{22} r_{2}+2 D_{66} r_{8}+2 D_{16} r_{5}+2 D_{26} r_{7}+4 D_{66} r_{9}$

$K_{i j}^{D}=P_{x} r_{10}+P_{x y}\left(r_{12}+r_{13}\right)+P_{y} r_{11}$

or in the non - dimensional form:

$K_{i j}=\frac{4 n^{3}}{m}\left(\frac{b}{a}\right) \bar{D}_{11} q_{1}+4 m n\left(\frac{a}{b}\right) \bar{D}_{12} q_{4}+4 n^{2} \bar{D}_{16} q_{6}+4 m n\left(\frac{a}{b}\right) \bar{D}_{12} q_{3}$

$+\frac{4 m^{3}}{n}\left(\frac{a}{b}\right) \bar{D}_{22} q_{2}+4 m^{2}\left(\frac{a}{b}\right)^{2} \bar{D}_{26} q_{8}+4 n^{2} \bar{D}_{16} q_{5}+4 m^{2}\left(\frac{a}{b}\right)^{2} \bar{D}_{26} q_{7}$

$+4 m n\left(\frac{a}{b}\right) \bar{D}_{66} q_{9}$

$K_{i j}^{D}=\bar{P}_{x} \frac{n}{m}\left(\frac{b}{a}\right) q_{10}+\bar{P}_{x y}\left(q_{12}+q_{13}\right)+\bar{P}_{y} \frac{m}{n}\left(\frac{a}{b}\right) q_{11}$

where

Also

$$
\bar{D}_{i j}=\left(\frac{1}{E_{1} h^{3}}\right) D_{i j}, \quad \bar{P}_{i}=\left(\frac{a}{E_{1} h^{3}}\right) P_{i}
$$

$$
\bar{w}=\left(\frac{1}{h}\right) w, \quad \bar{\phi}=\left(\frac{h}{a}\right) \phi, \quad \bar{\psi}=\left(\frac{h}{a}\right) \psi, \quad \bar{b}=b / a
$$

The transformed stiffnesses are as follows:

$$
\begin{aligned}
& C_{11}=C_{11}^{\prime} c^{4}+2 c^{2} s^{2}\left(C_{11}^{\prime}+2 C_{66}^{\prime}\right)+C_{22}^{\prime} s^{4} \\
& C_{12}=c^{2} s^{2}\left(C_{11}^{\prime}+C_{22}^{\prime}+4 C_{66}^{\prime}\right)+C_{12}^{\prime}\left(c^{4}+s^{4}\right) \\
& C_{16}=c s\left[C_{11}^{\prime} c^{4}+C_{22}^{\prime} s^{2}-\left(C_{12}^{\prime}+2 C_{66}^{\prime}\right)\left(c^{2}-s^{2}\right)\right] \\
& C_{22}=C_{11}^{\prime} s^{4}+2 c^{2} s^{2}\left(C_{12}^{\prime}+2 C_{66}^{\prime}\right)+C_{22}^{\prime} c^{4} \\
& C_{26}=c s\left[C_{11}^{\prime} s^{2}-C_{22}^{\prime} c^{2}-\left(C_{12}^{\prime}+2 C_{66}^{\prime}\right)\left(c^{2}-s^{2}\right)\right] \\
& C_{66}=\left(C_{11}^{\prime}+C_{22}^{\prime}-2 C_{12}^{\prime}\right) c^{2} s^{2}+C_{66}^{\prime}\left(c^{2}-s^{2}\right)^{2} \\
& \text { Where } \\
& C_{11}^{\prime}=\frac{E_{1}}{1-v_{12} v_{21}} \\
& C_{12}^{\prime}=\frac{v_{21} E_{1}}{1-v_{12} v_{21}}=\frac{v_{12} E_{1}}{1-v_{12} v_{21}} \\
& C_{22}^{\prime}=\frac{E_{2}}{1-v_{12} v_{21}} \\
& C_{44}^{\prime}=G_{23}, \quad C_{55}^{\prime}=G_{13} \quad \text { and } C_{66}^{\prime}=G_{12}
\end{aligned}
$$

$E_{1}$ and $E_{2}$ are the elastic moduli in the direction of the fiber and the transverse directions respectively, $v$ is the Suleiman, O. M. E., Osman, M. Y., \& Hassan, T. (2019). Effect of reversing lamination scheme. International Research Journal of Engineering, IT \& Scientific Research, 5(3), 28-42. https://doi.org/10.21744/irjeis.v5n3.645 
Poisson's ratio. $G_{12}, G_{13}$, and $G_{23}$ are the shear moduli in the $x-y$ plane, $y-z$ plane, and $x-z$ plane respectively, and the subscripts 1 and 2 refer to the direction of fiber and the transverse direction respectively.

\subsection{Effect of Aspect Ratio}

In order to study the stacking sequence of laminated plates, two lamination schemes of cross-ply (0/ 90) and (90/ $0)$ and two other laminations of angle-ply $(45 /-45)$ and $(-45 / 45)$ were considered. The results of their buckling loads of parameter $\left(\bar{P}=P a^{2} / E_{1} h^{3}\right)$ are given in Tables $1,2,3$ and 4 . Three boundary conditions SS, CC and CS are considered in this case. The buckling loads have been determined for two modulus ratios 40 and 5 . It is observed that, the buckling loads are completely the same for the given first three modes. Therefore, it can be concluded that the buckling load of laminated plates will remain the same even if the lamination order is reversed. The reason behind this is that the transformed elastic coefficients, $\left[C_{i j}\right]$, are equal for both lamination schemes.

Table 1

Non-dimensional buckling loads $\overline{\mathrm{P}}=\mathrm{Pa}^{2} / \mathrm{E}_{1} \mathrm{~h}^{3}$ of $(0 / 90)$ and $(90 / 0)$ lamination schemes of square laminated plates with $\mathrm{a} / \mathrm{h}=20$, and $\mathrm{E}_{1} / \mathrm{E}_{2}=40$

\begin{tabular}{lllll}
\hline \multirow{2}{*}{$\begin{array}{l}\text { Lamination } \\
\text { order }\end{array}$} & Mode & \multicolumn{3}{l}{ Boundary Conditions } \\
& Number & SS & CC & CS \\
\hline \multirow{3}{*}{$0 / 90$} & 1 & 0.4410 & 1.6885 & 1.1512 \\
& 2 & 0.4494 & 3.0311 & 1.6881 \\
& 3 & 1.4502 & 3.0349 & 2.5982 \\
$90 / 0$ & 1 & 0.4410 & 1.6885 & 1.1512 \\
& 2 & 0.4494 & 3.0311 & 1.6881 \\
& 3 & 1.4502 & 3.0349 & 2.5982 \\
\hline
\end{tabular}

Table 2

Non - dimensional buckling loads $\overline{\mathrm{P}}=\mathrm{Pa}^{2} / \mathrm{E}_{1} \mathrm{~h}^{3}$ of $(0 / 90)$ and $(90 / 0)$ lamination schemes of square laminated plates with $\mathrm{a} / \mathrm{h}=20$, and $\mathrm{E}_{1} / \mathrm{E}_{2}=5$

\begin{tabular}{lllll}
\hline Lamination & Mode & \multicolumn{3}{l}{ Boundary Conditions } \\
order & Number & SS & CC & CS \\
\hline \multirow{3}{*}{$0 / 90$} & 1 & 0.6970 & 2.2275 & 1.5593 \\
& 2 & 1.9943 & 3.9687 & 2.3388 \\
& 3 & 1.9954 & 3.9733 & 3.7581 \\
$90 / 0$ & 1 & 0.6970 & 2.2274 & 1.5594 \\
& 2 & 1.9944 & 3.9688 & 2.3393 \\
& 3 & 1.9957 & 3.9733 & 3.7580 \\
\hline
\end{tabular}

Table 3

Non - dimensional buckling loads $\overline{\mathrm{P}}=\mathrm{Pa}^{2} / \mathrm{E}_{1} \mathrm{~h}^{3}$ of (45/ -45) and (-45/45) lamination schemes of square laminated plates with $\mathrm{a} / \mathrm{h}=20$, and $\mathrm{E}_{1} / \mathrm{E}_{2}=40$

\begin{tabular}{lllll}
\hline $\begin{array}{l}\text { Lamination } \\
\text { order }\end{array}$ & Mode & \multicolumn{3}{l}{ Boundary Conditions } \\
& Number & SS & CC & CS \\
\hline \multirow{4}{*}{$45 /-45$} & 1 & 0.8375 & 1.6524 & 1.2806 \\
& 2 & 1.7263 & 2.7630 & 1.9965 \\
& 3 & 1.7285 & 2.7659 & 2.5358 \\
$-45 / 45$ & 1 & 0.8372 & 1.6527 & .2805 \\
& 2 & 1.7262 & 2.7631 & 19963 \\
& 3 & 1.7283 & 2.7660 & 2.5355 \\
\hline
\end{tabular}


Table 4

Non - dimensional buckling loads $\overline{\mathrm{P}}=\mathrm{Pa}^{2} / \mathrm{E}_{1} \mathrm{~h}^{3}$ of $(45 /-45)$ and $(-45 / 45)$ lamination schemes of square laminated plates with $\mathrm{a} / \mathrm{h}=20$, and $\mathrm{E}_{1} / \mathrm{E}_{2}=5$

\begin{tabular}{lllll}
\hline Lamination & Mode & \multicolumn{3}{l}{ Boundary Conditions } \\
order & Number & SS & CC & CS \\
\hline \multirow{3}{*}{$45 /-45$} & 1 & 0.9907 & 2.2010 & 1.6553 \\
& 2 & 2.1995 & 3.7613 & 2.5668 \\
& 3 & 2.2015 & 3.7652 & 2.4640 \\
$-45 / 45$ & 1 & 0.9908 & 2.2010 & 1.6553 \\
& 2 & 2.1995 & 3.7613 & 2.5671 \\
& 3 & 2.2015 & 3.7652 & 3.4636 \\
\hline
\end{tabular}

\section{Conclusion}

The finite element model has been formulated to compute the buckling loads of laminated plates with rectangular cross-section and to study the effect of reversing lamination scheme on the non - dimensional critical buckling loads of laminated composite plates. New results have been presented. The buckling loads have been determined for two modulus ratios 40 and 5. It is observed that the buckling loads are completely the same for the given first three modes. Therefore, it can be concluded that the buckling load of laminated plates will remain the same even if the lamination order is reversed. The reason behind this is that the transformed elastic coefficients, $\left[C_{i j}\right]$, are equal for both lamination schemes.

Conflict of interest statement and funding sources

The author(s) declared that (s)he/they have no competing interest.

\section{Statement of authorship}

The author(s) have a responsibility for the conception and design of the study. The author(s) have approved the final article.

\section{Acknowledgments}

The authors would like to acknowledge with deep thanks and profound gratitude Mr. Osama Mahmoud of Daniya Center for Publishing and Printing Services, Atbara, Sudan who spent many hours in editing, re-editing of the manuscript in compliance of IRJEIS.

Suleiman, O. M. E., Osman, M. Y., \& Hassan, T. (2019). Effect of reversing lamination scheme. International Research Journal of Engineering, IT \& Scientific Research, 5(3), 28-42. 


\section{References}

Al, A. W. (1986). khafaji and John R. Tooley,'Numerical methods in engineering practice', University of Evansville. Eastop, T. D., \& McConkey, A. (1986). Applied thermodynamics. Longman Group, London.

Elmardi, O. M. (2014). Verification of dynamic relax-ation method in isotropic, orthotropic and laminated plates using small deflection theory. International Journal of Advanced Science and Technology, 72(4), 37-48.

Larry, J. (1984). Segelind,'applied finite element analyses'. Agricultural engineering department, Michigan state University, John Wiley and sons publishers.

Suleiman, O. M. E. (2015). Nonlinear analysis of rectangular laminated plates. Lap Lambert Academic Publishing, Germany, and ISBN:(978-3-659-76787-6).

Suleiman, O. M. E. (2016). Bibliography and literature review on buckling of laminated plates. International Journal of Science and Engineering (EPH), 2(8), 104-112.

Suleiman, O. M. E. (2016). Introduction and Literature Review on Buckling of Composite Laminated Plates. Lap Lambert Academic Publishing, Germany, and ISBN:(978-3-659-86387-5), 5(10).

Suleiman, O. M. E. (2016). Text Book on Dynamic Relaxation Method. Lap Lambert Academic Publishing, Germany, and ISBN:(978-3-659-94751-3).

Suleiman, O. M. E. (2017). Deflection of laminated composite plates using dynamic relaxation method. International Journal of Physical Sciences and Engineering, 1(1), 40-53. https://doi.org/10.21744/ijpse.v1i1.5

Suleiman, O. M. E., Osman, M. Y., \& Hassan, T. (2019). Stability of thin laminated decks plates under plane compressive loading. International Research Journal of Engineering, IT \& Scientific Research, 5(2), 1-28. https://doi.org/10.21744/irjeis.v5n2.607

Turvey, G. J., \& Osman, M. Y. (1989). Large deflection analysis of orthotropic Mindlin plates'. In Proceedings of the 12th energy resources technical conference and Exhibition, Houston, Texas (pp. 163-172).

Turvey, G. J., \& Osman, M. Y. (1990). Elastic large deflection analysis of isotropic rectangular Mindlin plates. International journal of mechanical sciences,32(4), 315-328. https://doi.org/10.1016/00207403(90)90096-2

Turvey, G. J., \& Osman, M. Y. (1991). Large deflection effects in antisymmetric cross-ply laminated strips and plates. In Composite Structures (pp. 397-413). Springer, Dordrecht. https://doi.org/10.1007/978-94-011-3662431 


\section{Biography of Author}

\begin{tabular}{||l||}
\hline \hline \\
Osama Mohammed Elmardi Suleiman Khayal was born in Atbara, Sudan in 1966. He \\
received his diploma degree in mechanical engineering from Mechanical Engineering \\
College, Atbara, Sudan in 1990. He also received a bachelor degree in mechanical \\
engineering from Sudan University of science and technology - Faculty of engineering \\
in 1998, and a master degree in solid mechanics from Nile valley university (Atbara, \\
Sudan) in 2003, and a PhD in structural engineering in 2017. He contributed in teaching \\
some subjects in other universities such as Red Sea University (Port Sudan, Sudan), \\
Kordofan University (Obayed, Sudan), Sudan University of Science and Technology \\
(Khartoum, Sudan), Blue Nile University (Damazin, Sudan) and Kassala University \\
(Kassala, Sudan). In addition, he supervised more than hundred and fifty under \\
graduate studies in diploma and B.Sc. levels and about fifteen master theses. The author \\
wrote about thirty-five engineering books written in Arabic language, and fifteen books \\
written in English language and more than hundred research papers in fluid mechanics, \\
thermodynamics, internal combustion engines and analysis of composite structures. He \\
is currently an associated professor in Department of Mechanical Engineering, Faculty \\
of Engineering and Technology, Nile Valley University Atbara, Sudan. His research \\
interest and favorite subjects include structural mechanics, applied mechanics, control \\
engineering and instrumentation, computer aided design, design of mechanical \\
elements, fluid mechanics and dynamics, heat and mass transfer and hydraulic \\
machinery. The author also works as a technical manager and superintendent of Al - \\
Kamali mechanical and production workshops group which specializes in small, \\
medium and large automotive overhaul maintenance and which situated in Atbara town \\
in the north part of Sudan, River Nile State. \\
\hline
\end{tabular}

Suleiman, O. M. E., Osman, M. Y., \& Hassan, T. (2019). Effect of reversing lamination scheme. International Research Journal of Engineering, IT \& Scientific Research, 5(3), 28-42. https://doi.org/10.21744/irjeis.v5n3.645 